\title{
Intravital Imaging of Human Prostate Cancer Using Viral Nanoparticles Targeted to Gastrin-Releasing Peptide Receptors
}

\author{
Dr. Nicole F. Steinmetz ${ }^{\dagger,+}$, \\ Department of Cell Biology, Center of Integrative Molecular Biosciences, The Scripps Research \\ Institute, North Torrey Pines Rd., La Jolla, CA 92037, USA
}

Amber L. Ablack ${ }^{\dagger}$, Translational Prostate Cancer Research Group, London Regional Cancer Program, Room A4-823, 790 Commissioners Rd. E., London, Ontario N6A 4L6, Canada

Jennifer L. Hickey ${ }^{\dagger}$ Department of Chemistry, University of Western Ontario, London, Ontario N6A 5C1, Canada

Jailal Ablack, Department of Oncology, London Regional Cancer Program, 790 Commissioners Rd. E., London, Ontario N6A 4L6, Canada

Dr. Bhavik Manocha,

Translational Prostate Cancer Research Group London Regional Cancer Program, Room A4-823, 790 Commissioners Rd. E., London, Ontario N6A 4L6, Canada

\section{Dr. Joe S. Mymryk,}

Department of Oncology, London Regional Cancer Program, 790 Commissioners Rd. E., London, Ontario N6A 4L6, Canada

Dr. Leonard G. Luyt, and Department of Chemistry University of Western Ontario London, Ontario N6A 5C1, Canada

Dr. John D. Lewis

Translational Prostate Cancer Research Group London Regional Cancer Program, Room A4-823, 790 Commissioners Rd. E., London, Ontario N6A 4L6, Canada

\section{Abstract}

\author{
Multivalent nanoparticles have several key advantages in terms of solubility, binding avidity, and \\ uptake, making them particularly well suited to molecular imaging applications. Herein is reported \\ the stepwise synthesis and characterization of NIR viral nanoparticles targeted to gastrin-releasing \\ peptide receptors that are over-expressed in human prostate cancers. The pan-bombesin analogue, \\ [ $\beta$-Ala11, Phe13, Nle14]bombesin-(7-14), is conjugated to cowpea mosaic virus particles \\ functionalized with an NIR dye (Alexa Fluor 647) and polyethylene glycol (PEG) using the \\ copper(I)-catalyzed azide-alkyne cycloaddition reaction. Targeting and uptake in human PC-3 \\ prostate cells is demonstrated in vitro. Tumor homing is observed using human prostate tumor \\ xenografts on the chicken chorioallantoic membrane model using intravital imaging. Further

\footnotetext{
(c) 2011 Wiley-VCH Verlag GmbH \& Co. KGaA, Weinheim

Phone: 1-519-685-8600 x57194, Fax: 1-866-426-6697, John.Lewis@lhsc.on.ca.

${ }^{+}$Current address: Department of Biomedical Engineering, Case Center for Imaging Research, Case Western Reserve University, 11100 Euclid Avenue, Cleveland, OH 44106, USA

${ }^{\dagger}$ These authors contributed equally to this paper.

Supporting Information

Supporting Information is available from the Wiley Online Library or from the author.
} 
development of this viral nanoparticle platform may open the door to potential clinical noninvasive molecular imaging strategies.

\section{Introduction}

Prostate cancer is the most common malignancy among men, accounting for $10 \%$ of all cancer-related deaths in 2008. ${ }^{[1]}$ Conventional imaging, which includes computed tomography (CT), Magnetic Resonance Imaging (MRI), and ultrasound, is currently used to detect organ-confined or metastatic disease for staging and determining prognosis. However, there is substantial room for improvement in the specificity and sensitivity of imaging for detecting small primary lesions and for identifying minimal, metastatic disease. ${ }^{[2]}$

Nanoparticles are well suited for molecular imaging approaches because they can simultaneously display targeting ligands, imaging moieties, and other functional groups. ${ }^{[3]}$ Multivalency in terms of imaging moiety promotes high sensitivity for in-vivo detection of rare targets. ${ }^{[4]}$ Multivalency in terms of the targeting ligand ensures specificity and a high affinity for the desired target. ${ }^{[5]}$ Viral nanoparticles (VNPs) have recently gained attention because they are naturally occurring bio-nanomaterials that have several advantages over man-made synthetic nanoparticles such as dendrimers, quantum dots, or liposomes: i) VNPs are composed of degradable biomaterials, ii) VNPs derived from bacteriophages and plant viruses are considered safe from a human health perspective, iii) their structures are known to atomic resolution, allowing for ligand attachment with a high degree of structural control, and iv) particles can be predictably modified using chemical bioconjugation and genetic engineering, giving significant flexibility in their construction.

We have previously demonstrated that VNPs based on the cowpea mosaic virus (CPMV) can be used for long-term intravital imaging of vascular endothelium in embryos and tumors, ${ }^{[6]}$ and have described an approach that allows tunable densities of targeting ligand, detection moieties, and polyethylene glycol (PEG) to be incorporated into a single VNP. ${ }^{[4]}$ In this previous work, targeting ligands were conjugated directly to the VNP surface and adjacent to the PEG chains, which can result in partial ligand shielding, dependent upon the density and length of the PEG chains. ${ }^{[4,7]}$ Here, we sought to both improve the display of targeting ligands on the surface of CPMV VNPs, and to establish a methodology that would result in more stable nanoparticles when hydrophobic targeting ligands were used.

In this work, we report the stepwise synthesis and characterization of NIR viral nanoparticles targeted to gastrin-releasing peptide (GRP) receptors via the targeting peptide bombesin. Receptors for GRP are over-expressed in a variety of human tumors, including prostate cancer, and our previous work has shown that bombesin-functionalized iron oxide nan-oparticles are specifically taken up by prostate cancer cells in vitro. ${ }^{[8]}$ A novel VNP synthesis strategy is employed whereby the bombesin peptide ligands are attached at the outer ends of the PEG chains, distal to the VNP surface. Furthermore, we use copper(I)catalyzed azide-alkyne cycloaddition for both PEG and ligand attachment, in order to establish a complementary methodology to previous approaches, and to maximize the conjugation efficiency of hydrophobic ligands such as bombesin. Alkynes are introduced onto the VNP surface to serve as a ligation handle for azide-containing PEG-bombesin (targeted) or PEG (nontargeted) constituents. The resulting multifunctional VNPs display bombesin peptides, polyethylene glycol, and the NIR dye Alexa Fluor 647 (AF647). The PEG chains are introduced for two reasons: i) to reduce the immunogenicity of the particles, ${ }^{[9]}$ ii) to reduce nondesired cell interactions in vitro and in vivo, ${ }^{[6,7]}$ and iii) to enhance plasma circulation time. ${ }^{[10]} \mathrm{We}$ found also that linking the PEG chains to the 
targeting peptide increases the solubility of the peptide complex, resulting in an enhancement of bioconjugation efficiency.

Bombesin is a 14 amino acid peptide originally isolated from the skin of the fire-bellied frog, Bombina bombina. ${ }^{[11]}$ Radiolabelled analogues of bombesin specifically target cellsurface GRP receptors. ${ }^{[12-14]}$ Four receptor subtypes have been identified to date; GRP-R, NMB-R, BRS-3, and BB4-R. A very potent universal pan-bombesin ligand with a structure of [D-Tyr6, $\beta$-Ala11, Phe13, Nle14]bombesin-(6-14) $)^{[15]}$ has been shown to bind to all four GRP receptor subtypes with high affinity. To design a bombesin peptide suitable for attachment to CPMV, we removed the D-Tyr in position six (Figure 1A), which has been shown to have no significant effect on binding interactions with GRP receptors. ${ }^{[16,17]}$ The peptide was designed for attachment using its $N$-terminus, as radiolabelled bombesin conjugates containing extensive modifications to the $N$-terminus still retain a high affinity for their desired receptors. ${ }^{[14,18-20]}$ The $[\beta$-Ala11, Phe13, Nle14] bombesin-(7-14) peptide was further modified to include a second $\beta$-alanine extension in position six, followed by a PEG linker, to ensure that the active region of the ligand would be situated away from the virus particle. An $N$-terminal azide substituent was installed to allow for incorporation onto the VNPs through a copper-catalyzed azide-alkyne cycloaddition (click) reaction.

The ability of these NIR PEGylated VNPs displaying bombesin targeting ligands to specifically bind to human prostate cancer cells expressing the GRP receptor was assessed. The tumor-homing efficiency of these VNPs was then evaluated by intravital imaging in a xenograft chicken chorioallantoic membrane model of human prostate cancer.

\section{Results}

\subsection{Synthesis and Characterization of GRP Receptor-Targeted Viral Nanoparticles}

To covalently decorate CPMV with AF647, PEG, and the targeting ligands (azide-activated PEG-bombesin), a combination of standard lysine (Lys) coupling procedures using $N$ hydroxysuccinimide (NHS)-activated esters and click chemistry was used. Functionalities were installed on addressable Lys side chains on the solvent-exposed exterior surface of the viral capsid (Figure 1C). ${ }^{[21]}$ Dye-labeled CPMV-PEG and CPMV-PEG-bombesin were synthesized in three steps as outlined in Figure 1B. First, fluorescent dyes were attached using an NHS ester of AF647 in a limited stoichiometry to ensure only partial coverage. We then installed NHS-alkyne ( $N$-(4-pentynoyloxy) succinimide) on the remaining Lys side chains to provide a ligation handle for the click reaction that allows coupling of the azideactivated PEG-bombesin or PEG (third step). Click reactions were carried out using an optimized protocol. ${ }^{[22]}$

Samples from each conjugation step were purified and characterized first by native gel electrophoresis and size-exclusion chromatography. Both methods confirmed that the particles remained intact upon chemical modification and native gels indicated that dyes, PEG, and PEG-bombesin were indeed covalently attached (Figure 2A,B). The mobility of CPMV in the native gels is influenced by various factors including charge and hydrodynamic radius. ${ }^{[23]}$ The attachment of small chemical modifiers such as AF647 and alkyne to Lys side chains leads to an increased mobility of the VNPs in the matrix gel (Figure 2A). AF647-labeled samples were visible under white light, further confirming the presence of the dye.

Quantitation of dyes per CPMV was carried out using UV-visible spectroscopy and the specific extinction coefficient for the AF647. It was found that, after a reaction time of 4-5 h, around 50-60 labels were displayed per VNP. A coumarin assay was used to quantify the number of free alkynes. ${ }^{[24]}$ A nonfluorescent 3-azidocoumarin was utilized and added to 
CPMV-AF647-alkyne, CPMV-AF647-PEG-bombesin, and CPMV-AF647-PEG under click conditions. Coupling leads to the formation of a fluorescent triazolylcoumarin, which can be followed at $475 \mathrm{~nm}$. Based on an internal standard (propargyl alcohol at a known concentration) the number of free alkynes was quantified and it was found that around 80 95 alkynes were attached to CPMV-AF647. No free alkynes could be detected in the final products (CPMV-AF647-PEG-bombesin and CPMV-AF647-PEG), indicating that all free alkynes were utilized in the reaction and hence 80-95 PEG-bombesin and PEG molecules, respectively, were displayed per VNP formulation.

Size-exclusion chromatography (Figure 2B) was used to confirm the integrity of CPMVPEG-bombesin and CPMV-PEG. Both the elution time and the ratio of the absorbance peak at $260 \mathrm{~nm}$ (derived from the encapsidated RNA molecules) to the absorbance peak at 280 $\mathrm{nm}$ (derived from the protein coat) were in good agreement with data typically obtained for intact CPMV particles: intact CPMV elutes at 25.4 min with A260:280 $=1.8$ (not shown). The absorbance peak at $650 \mathrm{~nm}$ (derived from the AF647 dye) co-elutes with the 260 and $280 \mathrm{~nm}$ peaks, confirming that the dyes were stable and covalently attached. The diameter of the conjugated CPMV particles, as measured by dynamic light scattering (Figure 2C), was slightly larger than that typically obtained for intact CPMV particles $(29-30 \mathrm{~nm}){ }^{[25]}$ which would explain their reduced mobility in the native gel. ${ }^{[7]}$ Transmission electron microscopy (TEM) further confirmed the integrity of CPMV-PEG-bombesin and CPMV-PEG nanoparticles (Figure 2D).

\subsection{Uptake of CPMV-PEG-bombesin in Human Prostate Cancer Cells In Vitro}

To evaluate the ability of VNPs to specifically target GRP receptor-expressing cells, we treated human PC-3 prostate cancer cells with CPMV-PEG-bombesin and CPMV-PEG and visualized their binding and uptake by confocal microscopy. To allow us to visualize these cells using fluorescence microscopy in vitro and in vivo, we created a PC-3 cell line that stably expresses GFP in the cytoplasm. Human PC-3 cells express approximately 47600 GRP receptors per cell, ${ }^{[26]}$ and they are a well-established model for GRPR targeting and biological studies. ${ }^{[27,28]}$ CPMV-PEG did not bind PC-3 tumor cells at an appreciable level (Figure 3A, top panels), while CPMV-PEG-bombesin showed significant binding and uptake (Figure $3 \mathrm{~A}$, middle panels). To confirm the specificity of the observed binding, we performed a receptor block experiment where a 10-fold excess of unlabeled bombesin peptide was incubated with the cells for 10 min prior to incubation with CPMV-PEGbombesin. This resulted in a dramatic decrease in binding and uptake of the AF647-labeled CPMV-PEG-bombesin (Figure 3A, bottom panels), establishing that uptake is due to the specific interaction of CPMV-PEG-bombesin with GRP receptors.

To further confirm the uptake and specificity of GRP receptor-targeted VNPs, human PC-3 prostate cancer cells were treated with CPMV-PEG-bombesin and CPMV-PEG and uptake was quantitated by flow cytometry (Figure 3B,C). The binding and uptake of CPMV-PEGbombesin by PC-3 cells was significantly higher $(\mathrm{P}<0.05)$ than that of CPMV-PEG. When the receptors were blocked with a 10 -fold excess of unlabeled bombesin peptide, a statistically significant decrease $(\mathrm{P}<0.01)$ in binding and uptake was observed, reducing uptake to the level of CPMV-PEG alone. The level of nonspecific uptake of CPMV-PEG was higher than expected based on previous studies using this density and length of PEG, ${ }^{[7]}$ and may be the result of incomplete shielding of the native binding sites on the surface of the CPMV capsid. ${ }^{[10]}$

\subsection{Intravital Imaging of CPMV-PEG-Bombesin Uptake in Prostate Tumors}

To determine whether GRP receptor-targeted VNPs would localize to tumors in vivo, we utilized a previously established model for visualizing angiogenesis, ${ }^{[6]}$ tumor growth, and 
metastasis ${ }^{[29]}$ using noninvasive intravital fluorescence imaging. GFP-positive human PC-3 cells were implanted into the chorioallantoic membrane of the shell-less avian embryo and allowed them to grow into well-vascularized tumors averaging $5 \mathrm{~mm}$ in diameter. Using a specialized avian embryo incubator and spinning-disk confocal microscopy, the tumor was visualized via green fluorescent protein (GFP, $488 \mathrm{~nm}$ solid-state laser excitation, emission filter HQ535/50m) and the NIR channel to monitor AF647 (642 nm solid-state laser excitation, emission filter HQ700/50m). The main advantage of utilizing NIR fluorescent dyes such as AF647 is their exceptional tissue penetration and low tissue autofluorescence. Indeed, when the tumors were imaged prior to injection of the viral nanoparticles, no background signal was seen in the NIR channel (Figure 4A, leftmost panel). Upon intravenous injection of $50 \mu \mathrm{g}$ of CPMV-PEG-bombesin, a rapid initial uptake at the tumor site and the surrounding vasculature was observed during the first hour (Supporting Information (SI), Figure 2). During the next hour, the signal in the surrounding vasculature returned to background levels while the tumor signal was retained (Figure 4A). Over a period of $6 \mathrm{~h}$, a gradual accumulation of nanoparticles was observed within the tumor (Figure 4A). The distribution of signal within the tumor was not uniform, with a concentration of signal at the center of the tumor. When $50 \mu \mathrm{g}$ of CPMV-PEG nanoparticles was injected in a tumor-bearing avian embryo, a rapid increase in fluorescence both in the tumor and in the surrounding vasculature was also observed. In contrast to the targeted VNPs, the CPMV-PEG signal was rapidly washed out of the tumor and fluorescence remained low throughout the remainder of the experiment (Figure 4B and SI, Figure 3).

A statistically significant difference in prostate tumor uptake between targeted and control VNPs was observed at $2 \mathrm{~h}$ post-injection and thereafter $(\mathrm{P}<0.001)$ (Figure $4 \mathrm{C})$. This was further supported by the analysis of tumor sections from CPMV-PEG and CPMV-PEGbombesin treated animals (Figure 4D). Detection of VNPs using an anti-CPMV antibody demonstrates that bombesin-functionalized VNPs localized throughout the tumor tissue, while very little of the nontargeted CPMV was observed in the tumor. These data suggest that bombesin-functionalized CPMV nanoparticles would be suitable for targeted molecular imaging of GRP receptor over-expressing prostate tumors.

\section{Discussion}

\subsection{Considerations for In-Vivo Molecular Imaging with Nanoparticles}

A key challenge in the development of 'smart' molecular targeted tools for biomedical imaging is the capacity to selectively attach and display multiple functionalities on the nanoparticle platform. Here we have chosen the well-characterized plant virus CPMV. CPMV nanoparticles have been utilized for a wide range of applications spanning the fields of materials to medicine and the chemistry of the particles is well understood. ${ }^{[30]}$ To facilitate the decoration of CPMV virions with imaging and targeting molecules, a combination of standard NHS-coupling and click chemistry cycloadditions was used. CPMV displays 300 addressable Lys side chains on its exterior solvent-exposed surface, ${ }^{[21]}$ which can all be utilized under forcing conditions (i.e., with a high excess of coupling reagents, $\mathrm{pH}$ $>8.0$, and long incubation times). In this study, NIR AF647 fluorescent dyes were attached using NHS coupling. To facilitate partial labeling, a reduced concentration of dye was used and the reaction was stopped before completion after $4-5 \mathrm{~h}$, which yielded particles displaying 50-60 dyes. This allows for exceptional sensitivity for in-vivo intravital imaging, while the incomplete labeling permits the additional modification of the VNPs by utilizing free Lys side chains.

NHS-based chemistries are slow due to hydrolysis of the NHS ester in aqueous buffer solutions; however, it is a useful strategy for attaching small chemical modifiers such as fluorescent dyes. When attaching higher-molecular-weight molecules such as proteins or 
PEG and PEG-peptide conjugates, click reactions have been found to be more feasible ${ }^{[22,31]}$ due to their reduced requirement for reagent excess and shorter incubation times with higher loadings.

To achieve coupling of PEG-bombesin and PEG conjugates to CPMV, we first installed alkynes on Lys side chains which function as a ligation handle to allow coupling using the click cycloaddition. An average of 85-90 alkynes were installed, which means we functionalized $45-50 \%$ of the CPMV's available Lys side chains. We determined that $100 \%$ of the attached alkynes were utilized in the click reaction, which yielded particles displaying 85-90 PEG-bombesin ligands and PEG chains, respectively. We elected to install the targeting ligand via a PEG linker for two reasons. First, the bombesin peptide is a hydrophobic molecule and preliminary studies showed that direct attachment of this peptide to CPMV without a linker, as in our previously reported targeting study ${ }^{[4]}$ leads to instability and aggregation of the VNPs. This necessitated that all formulations had to be used freshly prepared, so our goal for this study was to design a stable particle formulation that would be better optimized for potential downstream applications. We hypothesized that by including a hydrophilic PEG linker into the hydrophobic bombesin peptide, we would achieve higher solubility and thus improved nanoparticle stability. Indeed, we found that CPMV-PEG-bombesin particles were stable and aggregation was not observed, as confirmed by native gel electrophoresis, size-exclusion chromatography, dynamic light scattering and TEM (Figure 2).

Although CPMV is a plant pathogen, it will bind and be internalized by endothelial cells in vivo. ${ }^{[6]}$ Interaction of CPMV with the mammalian vasculature is mediated primarily via a cell-surface form of the intermediate filament vimentin. ${ }^{[32,33]}$ Therefore, to achieve efficient targeting, it is necessary to shield these natural interactions using hydrophilic polymers such as PEG. Previous studies have shown that PEGylation is an effective strategy to minimize these undesired interactions. ${ }^{[6,7,34]}$ The shielding efficiency is highly dependent on the surface coverage achieved. ${ }^{[7,34]}$ Based on our previous studies, decoration of CPMV with greater than 80 PEG500 molecules ${ }^{[4]}$ or greater than 60 PEG2000 molecules ${ }^{[7]}$ is sufficient to allow effective shielding. In this study we found that when 80-95 PEG500 chains per VNP were attached, there was still a detectable level of nonspecific binding of the VNPs to PC-3 cells (Figure 3). Despite this, bombesin conferred an additional specific binding which was blocked in the presence of excess unlabelled peptide. Importantly, our in-vivo experiments indicate that this nonspecific binding did not significantly impact the tumor targeting conferred by the bombesin peptides (Figure 4).

Our intravital imaging studies using the chicken chorioallantoic membrane (CAM) tumor model showed that bombesin-targeted nanoparticles accumulate in human PC-3 tumors, and we were able to visualize and quantify the uptake of VNPs in the tumor in real time. We found that in contrast to the CPMV-PEG nanoparticles, CPMV-PEG-bombesin nanoparticles accumulated and were retained at significantly higher levels in tumors over extended periods of time, displaying relatively uniform uptake throughout the tumor. A low level of CPMV-PEG uptake at the core of tumors in vivo was also observed. This slow accumulation of nanoparticles in the center of the tumor in both targeted and nontargeted nanoparticles (Figure 4) is likely due to the enhanced permeation and retention (EPR) effect, which is a well-documented mechanism of passive tumor targeting by nanoparticles and liposomes. ${ }^{[35,36]}$ The EPR effect is the result of increased vascular permeability and inefficient lymphatic drainage in tumors, which allows sufficiently small particles to cross the endothelial fenestrations and accumulate in the tumor parenchyma. ${ }^{[35]}$ We have previously shown that while CPMV nanoparticles do not extravasate in healthy blood vessels, they will extravasate in tumors. ${ }^{[4]}$ Indeed, we surmise that efficient targeting and 
retention of CPMV-PEG-bombesin in PC-3 tumors is dependent upon extravasation into the tumor stroma via the EPR effect.

\section{Conclusion}

These studies demonstrate that CPMV-PEG-bombesin is a promising molecular imaging agent. Our observations that VNPs accumulate in very small tumors $(5 \mathrm{~mm})$ in the CAM model support the notion that the EPR effect occurs in small tumors and metastatic lesions, and that it can be exploited to achieve efficient tumor targeting with nanoparticles. For visualization, the NIR fluorescent dye AF647 was used due to its tissue penetration characteristics and low tissue autofluorescence in this wavelength range. Despite the favorable characteristics of NIR fluorescent agents, their utility for whole body imaging is limited. For the future application of CPMV-based VNPs for noninvasive molecular imaging, it will be necessary to incorporate alternative imaging moieties such as radioactive isotopes and MRI contrast agents, and evaluate their behavior in xenograft mouse models. Our data indicate that VNPs targeted to GRP receptors may be useful for the detection and visualization of human prostate cancer.

\section{Experimental Section}

\section{Propagation of CPMV}

Propagation and purification of CPMV was performed by standard procedures. ${ }^{[37]}$ The concentration of purified virions was determined photometrically at a wavelength of $260 \mathrm{~nm}$ using the molar extinction coefficient of $\varepsilon=8.1 \mathrm{~mL} \mathrm{mg}^{-1} \mathrm{~cm}^{-1}$.

\section{Bombesin Peptide Synthesis}

The fully-protected resin-bound peptide was synthesized via standard Fmoc solid-phase peptide chemistry using an APEX 396 automated peptide synthesizer. Fmoc (9fluorenylmethoxycarbonyl)-protected rink amide 4-methylbenzhydrylamine (MBHA) resin (loading $0.47 \mathrm{meq} \mathrm{g}^{-1}$ ) was utilized as the solid support. All $N$-Fmoc amino acids were employed, with a $550 \mathrm{MW} \mathrm{N} \mathrm{N}_{3}-(\mathrm{PEG})_{7}-\mathrm{COOH}$ spacer used to install the azide functionality at the $\mathrm{N}$-terminus of the peptide. Fmoc removal was achieved by treatment with $20 \%$ piperidine in dimethylformamide (DMF) for 5 and 20 min with consecutive DMF and tetrahydrofuran (THF) washes after each treatment. For each Fmoc amino acid coupling, resin was treated twice with 3 eq. of Fmoc amino acids, 3 eq. of HBTU $(2-(1 \mathrm{H}-$ benzotriazole-1-yl)-1,1,3,3-tetramethylaminium hexafluorophosphate) and 6 eq. of DIPEA $(\mathrm{N}, \mathrm{N}$-diisopropylethylamine) in $2 \mathrm{~mL}$ of DMF for $30 \mathrm{~min}-2 \mathrm{~h}$. The resin was washed consecutively with DMF and THF following each coupling. A final Fmoc deprotection was

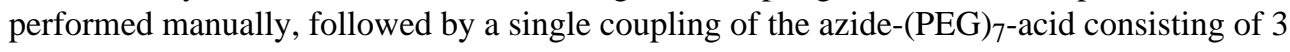
eq. of azide, 3 eq. of HBTU and 6 eq. of DIPEA in $4 \mathrm{~mL}$ of DMF for $2 \mathrm{~h}$.

Once the linear sequence was complete, the peptide was deprotected and cleaved from the resin by treatment with trifluoroacetic acid (TFA) containing water $(5 \% \mathrm{v} / \mathrm{v})$, phenol $(5 \% \mathrm{~m} /$ $\mathrm{v})$ and triisopropylsilane ( $2 \% \mathrm{v} / \mathrm{v})$ as scavengers for $4 \mathrm{~h}$. The resin was filtered and rinsed with a small amount of TFA. The resultant peptide was then precipitated from the TFA solution using tert-butyl methyl ether (TBME), and collected after centrifugation and decantation. The peptide was then rinsed with TBME and centrifugation and decantation was repeated. The resulting solid was redissolved in water and lyophilized to obtain the crude peptide. Purification was carried out by preparative high-performance liquid chromatography (HPLC, linear gradient of $25-70 \%$ solvent A in B) with the purity of the isolated white fluffy peptide determined to be $99 \%$ by analytical HPLC. Yield: $28.5 \mathrm{mg}$ (10\%). MS (ESI): $\mathrm{m} / \mathrm{z}$ calcd 1578.79, found $1579.36[\mathrm{M}+\mathrm{H}]^{+}$. 
Analytical HPLC was performed using a Grace Vydac Protein/Peptide RP-C18 column 4.6 $\mathrm{mm} \times 250 \mathrm{~mm}, 5 \mu \mathrm{m}$ (SI, Figure 1). Preparative HPLC was performed using a Grace Vydac Protein/Peptide RP-C18 column $22.0 \mathrm{~mm} \times 250 \mathrm{~mm}, 10 \mu \mathrm{m}$. A gradient system was used consisting of: $\mathrm{CH}_{3} \mathrm{CN}+0.1 \%$ of TFA (solvent A) and $\mathrm{H}_{2} \mathrm{O}+0.1 \%$ of TFA (solvent B) and the absorbance was detected at wavelengths of 220 and $254 \mathrm{~nm}$. The electrospray ionization mass spectrum was obtained using a Micromass Quatro Micro LCT mass spectrometer.

\section{Synthesis of Multifunctional CPMV Conjugates}

This is a three-step procedure: First, AF647 imaging molecules were covalently attached to solvent exposed Lys residues on CPMV using $N$-hydroxy-succinimide esters (Invitrogen, Carlsbad, CA). Second, an alkyne was introduced to remaining Lys side chains using $\mathrm{N}$-(4Pentynoyloxy) succinimide. Third, PEG and PEG-bombesin targeting ligands were attached using click chemistry.

Step 1: AF647 succinimidyl ester was dissolved in dimethyl sulfoxide (DMSO), the labels were used in a molar excess of 1000 to CPMV $\left(2-3 \mathrm{mg} \mathrm{mL}^{-1}\right)$, the reaction was carried out for $4-5 \mathrm{~h}$ at room temperature in the dark in a phosphate buffered saline (PBS):DMSO mixture of 9:1. Samples were purified by ultrapelleting (Beckman 50.2 Ti rotor, $42000 \mathrm{rpm}$, $\left.3 \mathrm{~h}, 4^{\circ} \mathrm{C}\right)$. CPMV was resuspended in PBS.

Step 2: $N$-(4-pentynoyloxy) succinimide (provided by Vu Hong, The Scripps Research Institute, TSRI) was added in a molar excess of 2000:1 per CPMV nanoparticle. Reaction conditions were as described above, after overnight reaction samples were purified by ultrapelleting and resuspended in PBS and stored at $4{ }^{\circ} \mathrm{C}$.

Step 3: Covalent attachment of PEG and PEG-bombesin was achieved using optimized click chemistry protocols. ${ }^{[22]}$ To a buffered solution of CPMV-A647-alkyne $(0.4 \mu \mathrm{M}$ final concentration) azide-PEG-bombesin and azide-PEG-bombesin $(150 \mu \mathrm{M})$ in DMSO, respectively, was added in a molar excess of 625:1 per CPMV. For coupling the following reagents were added: amino guanidine (AMG, $5 \mathrm{mM}$ ), $\mathrm{CuSO}_{4}$ :TBTA-OH in a molar ratio of 1:5 (500 $\mu \mathrm{M} \mathrm{CuSO}_{4}, 2.5 \mathrm{mM}$ TBTA-OH), and sodium ascorbate (Sod Asc) $(5 \mathrm{mM})$. The ligand TBTA-OH (trishydroxypropyl triazole) was provided by Vu Hong (TSRI). The reaction mixture was incubated at room temperature for $60 \mathrm{~min}$ on a shaker prior to purification by ultrapelleting (as described above). Samples were resuspended in PBS and stored at $4^{\circ} \mathrm{C}$.

\section{Native and Denaturing Protein Gel Electrophoresis of CPMV Nanoparticle Conjugates}

$20 \mu \mathrm{g}$ of CPMV, CPMV-A647, CPMV-A647-alkyne, CPMV-A647-PEG, and CPMVA647-PEG-bombesin, in PBS (in loading dye, MBI Fermentas) were analyzed on 1.2\% (w/ v) agarose gels in $1 \times$ TBE buffer, running buffer was $0.5 \times$ TBE. Protein subunits were analyzed on denaturing 4-12\% NuPage gels (Invitrogen) using 1×MOPS buffer. $20 \mu \mathrm{g}$ of each VNP formulation in PBS (added LDS loading buffer; Invitrogen) were analyzed. After completion of the electrophoretic separation the particles and protein subunits were stained using Coomassie Blue. Photographs of the gels were taken using FluorChemSP imaging system.

\section{Size-Exclusion Chromatography of Native and Modified CPMV Nanoparticles}

CPMV, CPMV-A647, CPMV-A647-alkyne, CPMV-A647-PEG, and CPMV-A647-PEGbombesin were analyzed on a Sepharose6 size-exclusion column using the AKTA Explorer. $40 \mu \mathrm{g}$ in $500 \mu \mathrm{L} 0.1 \mathrm{M}$ potassium phosphate buffer $\mathrm{pH} 7.0$ were analyzed at a flow rate of $0.5 \mathrm{mg} \mathrm{mL}^{-1}$. 


\section{Quantification of A647 Labels Per CPMV Particle Using UV-Visible Spectroscopy}

Dyes were quantified using UV-visible spectroscopy and the specific extinction coefficient for the A647 dyes $\left(\varepsilon=23700 \mathrm{M}^{-1} \mathrm{~cm}^{-1}\right)$. UV-visible data were recorded using a Beckman Coulter DU 800 spectrophotometer.

\section{Quantification of Alkynes and Number of Attached Peptides Per CPMV Using a Coumarin Assay}

The fluorescence assay used to quantify the number of free alkynes per CPMV was adopted from a previously reported method. ${ }^{[24]}$ In brief, nonfluorescent 3-azidocoumarin was added to CPMV-A647-alkyne, CPMV-A647-PEG, and CPMV-A647-PEG-bombesin under 'click \ conditions as described above. Coupling of the nonfluorescent 3-azidocoumarin to an alkyne leads to the formation of a fluorescent triazolylcoumarin which can be followed at $475 \mathrm{~nm}$. Propargyl alcohol at a known concentration was used as an internal standard allowing quantification of free alkynes. The fluorescence assay was performed using a Varioskan Flash Fluorescence Reader (Thermo Electron Corporation) and reagents were provided by Vu Hong (TSRI).

\section{Dynamic Light Scattering}

Particle size was measured by dynamic light scattering (90PLUS, Brookhaven Instruments, $\mathrm{NY}$ ). Samples were diluted in $10 \mathrm{mM}$ potassium chloride to a final concentration of $0.01-$ $0.02 \mathrm{mg} \mathrm{mL}^{-1}$, and measured for at least $10 \mathrm{~min}, 1 \mathrm{~min}$ per run. Data represents the mean \pm s.d of 3 independent runs per sample.

\section{TEM}

A total of $2 \mathrm{uL}$ of $0.4 \mathrm{mg} \mathrm{mL}^{-1}$ of bombesin-CPMV or PEG-CPMV in PBS were added to copper coated grids. An equal volume of $2 \%$ uranyl acetate was added for negative staining for $1 \mathrm{~min}$ at room temperature. The grids were dried and imaged using a Philips EM 410 electron microscope.

\section{Uptake of CPMV Conjugates in PC-3 Cells}

To generate a PC-3 cell line ubiquitously expressing GFP, cultured cells were infected with pLXSN-GFP (Clontech) and selected by neomycin selection without clonal propagation. Selected cells were passed in vivo to maintain a tumorigenic phenotype. PC-3 cells were grown in F-12 medium (Invitrogen) supplemented with 10\% fetal bovine serum and 1\% penstrep at $37{ }^{\circ} \mathrm{C}$ in $5 \% \mathrm{CO}_{2}$. Cells were seeded at a concentration of $3.5 \times 10^{5}$ cells mL $\mathrm{mL}^{-1}$ in a 12-well dish containing sterile $18 \mathrm{~mm}$ glass coverslips and grown for $24 \mathrm{~h}$. Cells were then incubated with $1 \mu \mathrm{g}$ of different virus preparations for $2 \mathrm{~h}$ at $37^{\circ} \mathrm{C}$ in $5 \% \mathrm{CO}_{2}$. Blocking experiments were performed by adding $100 \mathrm{X}$ unlabeled bombesin peptide with $1 \mu \mathrm{g}$ of bombesin-PEG-AF647-conjugated CPMV and incubated for $2 \mathrm{~h}$ at $37{ }^{\circ} \mathrm{C}$ in $5 \% \mathrm{CO}_{2}$. Cells were then washed five times on a rocker to remove unbound virus. Cells were then fixed with $2 \%$ formaldehyde in PBS for 15 min. Cells were washed two times, and mounted on slides with Prolong Gold containing DAPI (Invitrogen) and imaged using confocal microscopy (Quorum WAVE spinning disk). PC-3 cells were seeded in a 6-well dish at $5 \times$ $10^{5}$ cells $\mathrm{mL}^{-1}$ and grown $24 \mathrm{~h}$. One microgram of each virus preparation was added to 1 $\mathrm{mL}$ of F-12 medium with supplements mentioned above to live cells and incubated at $37^{\circ} \mathrm{C}$ in $5 \% \mathrm{CO}_{2}$ for $2 \mathrm{~h}$. After four washes with PBS, cells were trypsinized. Cells were then washed two times with PBS containing 5\% fetal bovine serum. The samples were then measured with a FACScalibur instrument (BD) and the data was analyzed using FlowJo 7.5.2 software (Tree Star Inc., Ashland, OR). Single live cells were gated and at least 10000 events were collected. 


\section{Intravital Imaging of Human Prostate Tumors in the Avian Embryo}

Tumors were generated by microinjecting 50000 tumor cells as a bolus below the CAM surface in 10 day embryos and allowed to grow for 7 days. Embryos ( $n=10$ per group) were then injected with $50 \mu \mathrm{g}$ of CPMV conjugate and placed within the microscope embryo incubator. GFP (488 $\mathrm{nm}$ solid-state laser excitation, emission filter HQ535/50m) and NIR (642 nm solid-state laser excitation, emission filter HQ700/50m) channels were visualized by intravital spinning-disk confocal microscopy, using a specialized instrument (Quorum Technologies, Guelph, ON, Canada) comprised of an upright Zeiss AxioExaminer Z1, LUDL filter wheels and large format motorized stage, a Yokogawa spinning disk head and a Hamamatsu 9100-12 ImageEM CCD camera, controlled by Volocity (Improvision, UK). A Z-stack of images of the tumor and the surrounding vasculature was captured and flattened into a single image. Image stacks were collected and analyzed using the software packages OpenLab and Volocity (Improvision, Inc).

\section{Analysis of Frozen Sections}

$6 \mathrm{~h}$ after injection, tumors were excised from the embryo, washed in PBS, and placed in a prefix solution (10\% sucrose and $3.7 \%$ formalin in PBS) at $4{ }^{\circ} \mathrm{C}$ overnight to preserve the tumor GFP signal. Next, tumors were washed in PBS and frozen in OCT (Tissue Tek) on dry ice. Frozen sections $(8 \mu \mathrm{m})$ were prepared (Leica CM $3050 \mathrm{~S}$ cryostat) on histobond glass slides. Samples were then blocked using 10\% goat serum in PBS for $1 \mathrm{~h}$. Rabbit antiCPMV antibody was added in 5\% goat serum ( $2 \mathrm{~h}$, room temperature) and washed (PBS, 4 times). Goat anti-rabbit IgG AlexaFluor 555 antibody (Invitrogen) was added in 5\% goat serum $(1 \mathrm{~h})$ and mounted in Prolong Gold (Invitrogen) mounting media. Sections were imaged using confocal microscopy (Quorum WAVE spinning disk) with a 20× objective.

\section{Supplementary Material}

Refer to Web version on PubMed Central for supplementary material.

\section{Acknowledgments}

This study was supported by grant \#84535 from the Canadian Institutes of Health Research to J.D.L., NSERC Grant \#326972 to L.G.L., NIH K99 EB009105 and American Heart Association Postdoctoral Fellowship to N.F.S. We acknowledge useful discussions with M. Manchester (UCSD), and Vu Hong (TSRI) is thanked for providing coupling reagents for click chemistry.

\section{References}

1. Jemal A, Siegel R, Ward E, Hao Y, Xu J, Murray T, Thun MJ. CA Cancer J Clin. 2008; 58:71. [PubMed: 18287387]

2. Zaheer A, Cho SY, Pomper MG. J Nucl Med. 2009; 50:1387. [PubMed: 19690043]

3. Manchester M, Singh P. Adv Drug Deliv Rev. 2006; 58:1505. [PubMed: 17118484]

4. Brunel FM, Lewis JD, Destito G, Steinmetz NF, Manchester M, Stuhlmann H, Dawson PE. Nano Lett. 2010; 10:1093. [PubMed: 20163184]

5. Montet X, Funovics M, Montet-Abou K, Weissleder R, Josephson L. J Med Chem. 2006; 49:6087. [PubMed: 17004722]

6. Lewis JD, Destito G, Zijlstra A, Gonzalez MJ, Quigley JP, Manchester M, Stuhlmann H. Nat Med. 2006; 12:354. [PubMed: 16501571]

7. Steinmetz NF, Manchester M. Biomacromolecules. 2009; 10:784. [PubMed: 19281149]

8. Martin AL, Hickey JL, Ablack AL, Lewis JD, Luyt LG, Gillies ER. J Nanoparticle Res. 2009; 12:1599.

9. Raja KS, Wang Q, Finn MG. ChemBioChem. 2003; 4:1348. [PubMed: 14661279] 
10. Steinmetz NF, Cho CF, Ablack A, Lewis JD, Manchester M. Nanomedicine. 2011; 6:351. [PubMed: 21385137]

11. Anastasi A, Erspamer V, Bucci M. Experientia. 1971; 27:166. [PubMed: 5544731]

12. de Visser M, Bernard HF, Erion JL, Schmidt MA, Srinivasan A, Waser B, Reubi JC, Krenning EP, de Jong M. Eur J Nucl Med Mol Imaging. 2007; 34:1228. [PubMed: 17287960]

13. Ferro-Flores G, Arteaga de Murphy C, Rodriguez-Cortes J, Pedraza-Lopez M, Ramirez-Iglesias MT. Nucl Med Commun. 2006; 27:371. [PubMed: 16531924]

14. La Bella R, Garcia-Garayoa E, Langer M, Blauenstein P, Beck-Sickinger AG, Schubiger PA. Nucl Med Biol. 2002; 29:553. [PubMed: 12088725]

15. Reubi JC, Wenger S, Schmuckli-Maurer J, Schaer JC, Gugger M. Clin Cancer Res. 2002; 8:1139. [PubMed: 11948125]

16. Broccardo M, Falconieri Erspamer G, Melchiorri P, Negri L, de Castiglione R. Br J Pharmacol. 1975; 55:221. [PubMed: 1201380]

17. Girard F, Bachelard H, St-Pierre S, Rioux F. Eur J Pharmacol. 1984; 102:489. [PubMed: 6489436]

18. Van de Wiele C, Dumont F, Vanden Broecke R, Oosterlinck W, Cocquyt V, Serreyn R, Peers S, Thornback J, Slegers G, Dierckx RA. Eur J Nucl Med. 2000; 27:1694. [PubMed: 11105826]

19. Baidoo KE, Lin KS, Zhan Y, Finley P, Scheffel U, Wagner HN Jr. Bioconjug Chem. 1998; 9:218. [PubMed: 9548537]

20. Smith CJ, Sieckman GL, Owen NK, Hayes DL, Mazuru DG, Kannan R, Volkert WA, Hoffman TJ. Cancer Res. 2003; 63:4082. [PubMed: 12874010]

21. Chatterji A, Ochoa WF, Paine M, Ratna BR, Johnson JE, Lin T. Chem Biol. 2004; 11:855. [PubMed: 15217618]

22. Hong V, Presolski SI, Ma C, Finn MG. Angew Chem Int Ed. 2009; 48:9879.

23. Steinmetz NF, Evans DJ, Lomonossoff GP. ChemBioChem. 2007; 8:1131. [PubMed: 17526061]

24. Sivakumar K, Xie F, Cash BM, Long S, Barnhill HN, Wang Q. Org Lett. 2004; 6:4603. [PubMed: 15548086]

25. Steinmetz NF, Hong V, Spoerke ED, Lu P, Breitenkamp K, Finn MG, Manchester M. J Am Chem Soc. 2009; 131:17093. [PubMed: 19904938]

26. Aprikian AG, Han K, Chevalier S, Bazinet M, Viallet J. J Mol Endocrinol. 1996; 16:297. [PubMed: 8782088]

27. Maina T, Nock B, Mather S. Cancer Imaging. 2006; 6:153. [PubMed: 17098646]

28. Cornelio DB, Roesler R, Schwartsmann G. Ann Oncol. 2007; 18:1457. [PubMed: 17351255]

29. Zijlstra A, Lewis J, Degryse B, Stuhlmann H, Quigley JP. Cancer Cell. 2008; 13:221. [PubMed: 18328426]

30. Steinmetz NF, Lin T, Lomonossoff GP, Johnson JE. Curr Top Microbiol Immunol. 2009; 327:23. [PubMed: 19198569]

31. Sen Gupta S, Raja KS, Kaltgrad E, Strable E, Finn MG. Chem Commun. 2005:4315.

32. Koudelka KJ, Rae CS, Gonzalez MJ, Manchester M. J Virol. 2007; 81:1632. [PubMed: 17121801]

33. Koudelka KJ, Destito G, Plummer EM, Trauger SA, Siuzdak G, Manchester M. PLoS Pathog. 2009; 5:e1000417. [PubMed: 19412526]

34. Destito G, Yeh R, Rae CS, Finn MG, Manchester M. Chem Biol. 2007; 14:1152. [PubMed: 17961827]

35. Iyer AK, Khaled G, Fang J, Maeda H. Drug Discov Today. 2006; 11:812. [PubMed: 16935749]

36. Maeda H, Wu J, Sawa T, Matsumura Y, Hori K. J Control Release. 2000; 65:271. [PubMed: 10699287]

37. Wellink J. Meth Mol Biol. 1998; 81:205.

38. a) Sayle RA, Milner-White EJ. RasMol: Biomolecular Graphics for All. Trends Biochem Sci. 1995; 20:374. [PubMed: 7482707] b) Reddy VS, Natarajan P, Okerberg B, Li K, Damodaran KV, Morton RT, Brooks CL III, Johnson JE. Virus Particle Explorer (VIPER): a website for virus capsid structures and their computational analysis. J Virol. 2001; 75:11943. [PubMed: 11711584] 


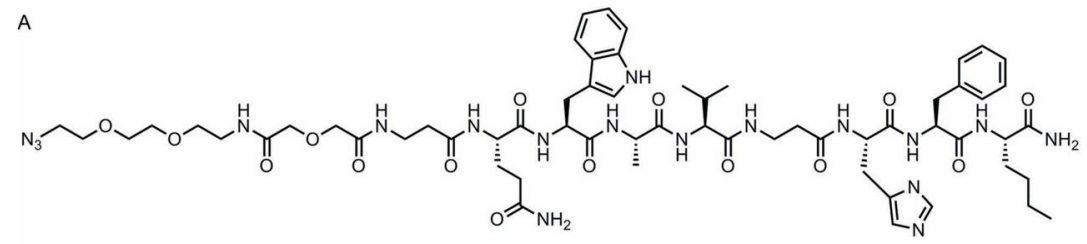

B

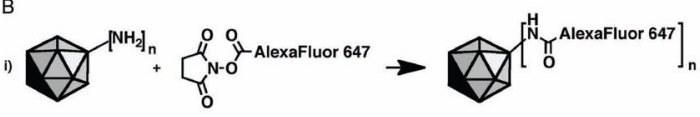

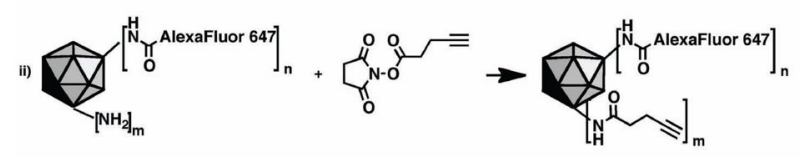
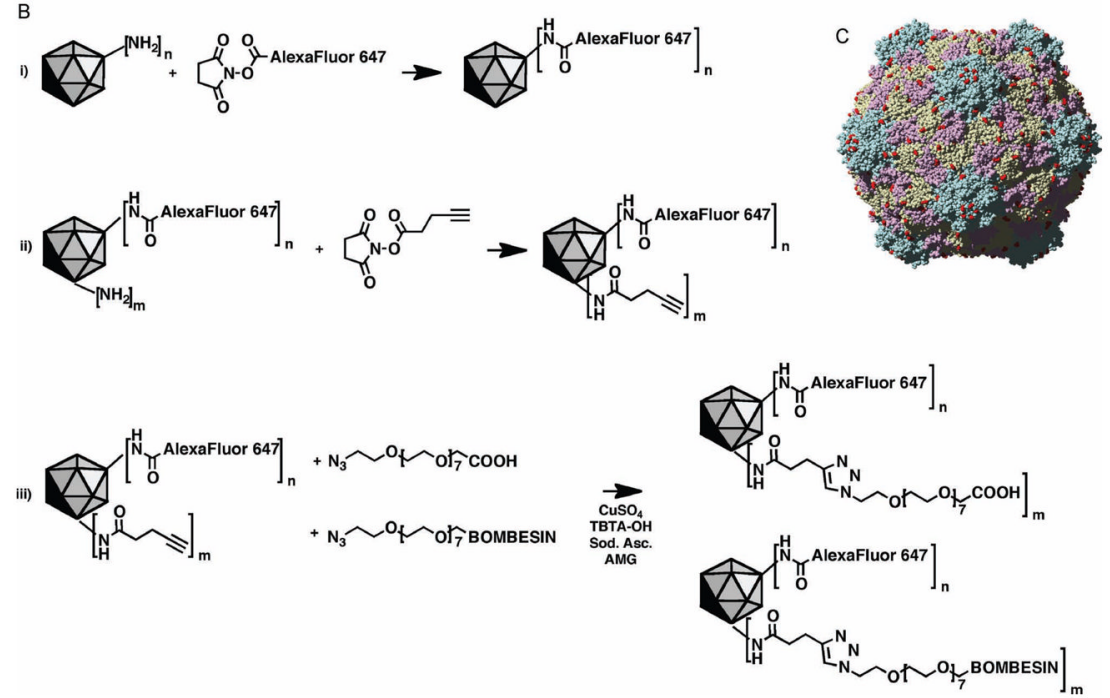

Figure 1.

Targeted viral nanoparticle synthesis strategy. A) Chemical structure of modified [ $\beta$-Ala11, Phe13, Nle14]bombesin-(7-14) peptide with a second $\beta$-alanine extension in position six followed by a PEG linker. B) Dye-labeled CPMV-PEG and CPMV-PEG-bombesin were synthesized in three steps; i) fluorescent dyes were attached using NHS-AF647 with partial coverage; ii) ( $N$-(4-pentynoyloxy)succinimide) was reacted with the remaining Lys side chains; iii) coupling of azide-activated PEG-bombesin or PEG alone by copper(I)-catalyzed azide-alkyne cycloaddition. Sod. Asc. is sodium ascorbate $(5 \mathrm{mM})$. C) Molecular model of CPMV nanoparticle with Lys sidechains highlighted in red. Image created with RasMol using oligomer coordinates generated by the VIPER website ${ }^{[38]}$ from protein data bank file 1 NY7. 
A

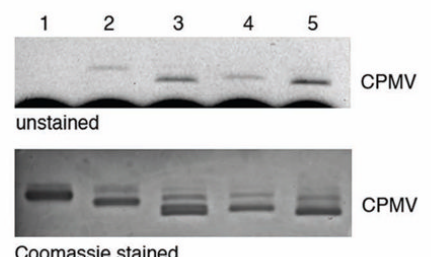

B

CPMV-A647-PEG
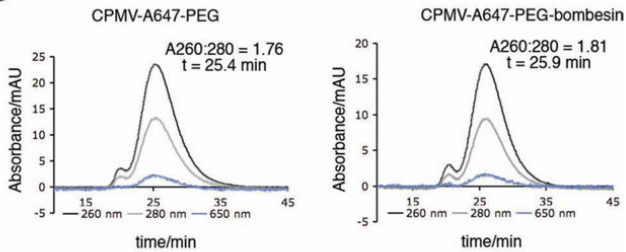

C

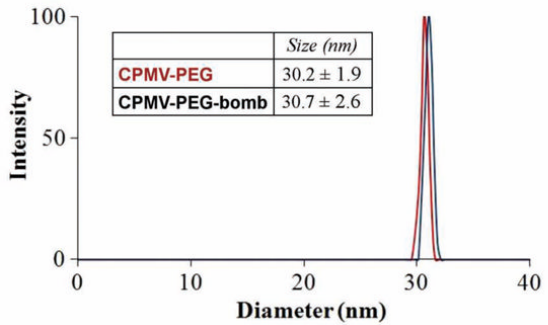

D

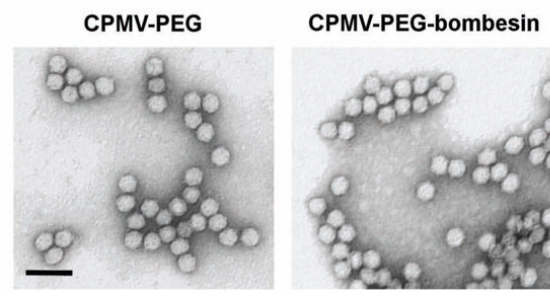

Figure 2.

Characterization of CPMV particles displaying fluorescent dyes, PEG, and peptides. A)

Native gel electrophoresis of intact CPMV particles (15 $\mu$ g, added loading dye) using a $1.2 \%$ $(w / v)$ agarose gel in $1 \times$ Tris/Borate/EDTA buffer (TBE) buffer. Dye-labeled particles were visualized as blue bands in the gel under white light (top panel), native and modified CPMV samples were also visualized after Coomassie staining (lower panel). Lanes are; $1=\mathrm{CPMV}$, 2 = CPMV-AF647, 3 = CPMV-AF647-alkyne, 4 = CPMV-AF647-PEG-bombesin, 5 = CPMV-AF647-PEG. B) Size-exclusion chromatography of CPMV-AF647-PEG-bombesin and CPMV-AF647-PEG using a Sepharose6 column. Shown is the absorbance versus elution time. CPMV was followed at an absorbance of $260 \mathrm{~nm}$ to detect the encapsidated RNA (black line) and at $280 \mathrm{~nm}$ detecting the protein coat (grey line), and at $650 \mathrm{~nm}$ to detect the attached AlexaFluor647 moieties (blue line). Inset: Elution time ( $t$ in min) as well as the absorbance ration of $260 / 280 \mathrm{~nm}$ is given. C) Particle sizes were measured by dynamic light scattering. Data (table inset) represents mean \pm the standard deviation (s.d.) of three independent runs per sample. Graph represents a single run of each, CPMV-PEG and CPMV-PEG-bombesin. D) TEM images of negative-stained CPMV conjugates. Scale bar = $60 \mathrm{~nm}$. 
A

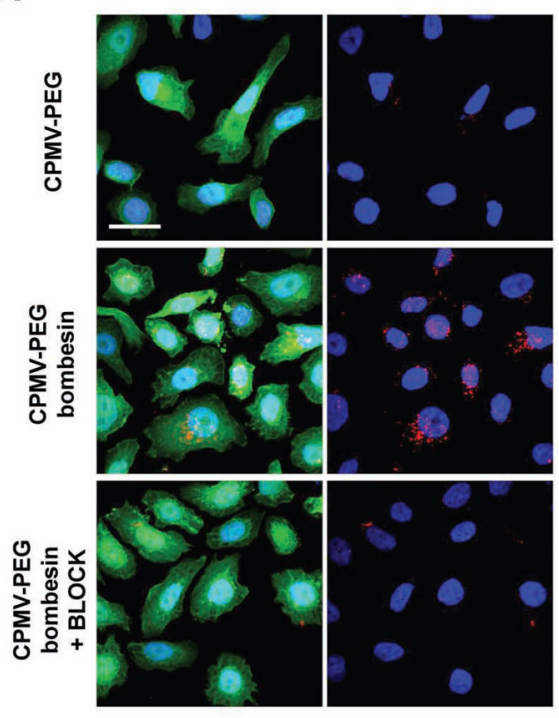

B

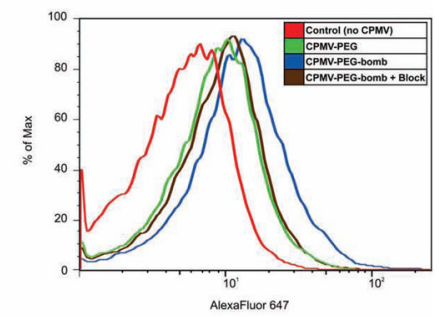

C

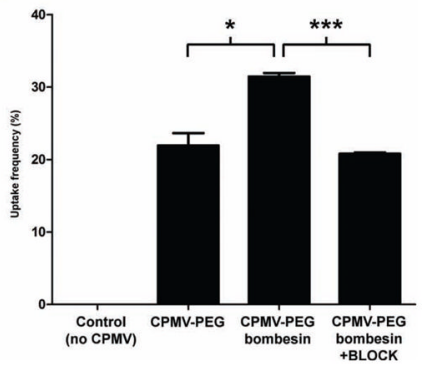

Figure 3.

Uptake of CPMV conjugates in human prostate cancer cells. A) Fluorescence confocal microscopy images of the uptake of CPMV-AF647 conjugates (red) into PC-3 GFP human prostate cancer cells (green). Nucleus is labeled with 4',6-diamidino-2-phenylindol (DAPI, blue). Scale bar $=15 \mu \mathrm{m}$. B) Histogram of AF647 fluorescence using flow cytometry of PC-3 tumor cells incubated with CPMV conjugates. C) Quantification of uptake of CPMV conjugates into PC-3 cells using flow cytometry. Mean percentage of AF647 positive cells \pm standard error of the mean (SEM) is shown for each treatment. Single live cells were gated and at least 10000 events were collected. CPMV-PEG-bombesin is taken up more efficiently than CPMV-PEG $(\mathrm{P}<0.05)$, and this is effectively blocked by an excess of free unlabeled peptide $(\mathrm{P}<0.001)$. 
A

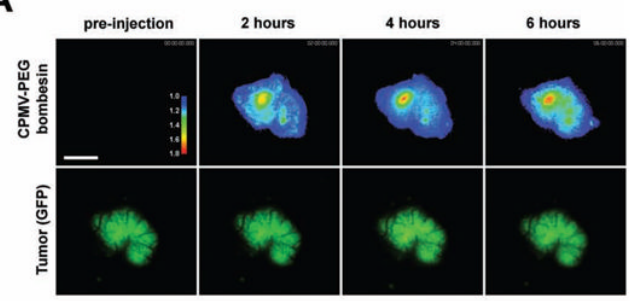

B

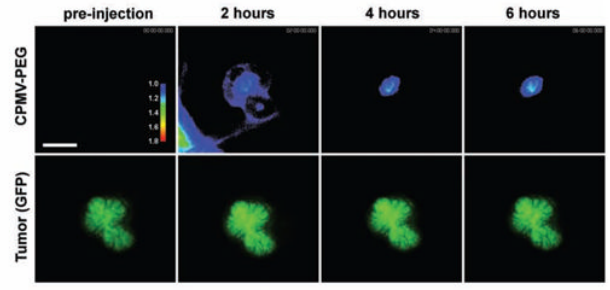

C
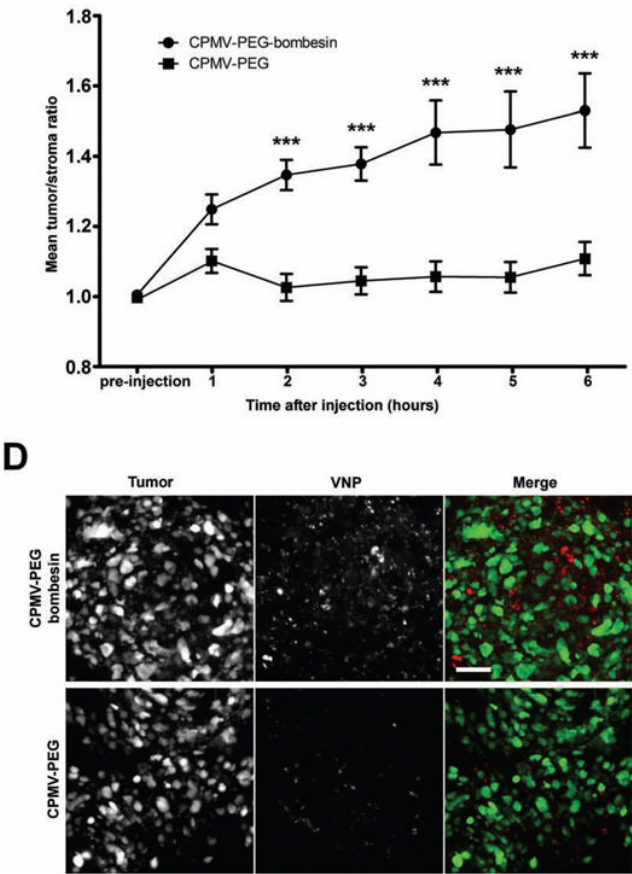

Figure 4.

Intravital imaging of viral nanoparticle uptake in prostate tumors in vivo. A) Intravital fluorescence confocal imaging of PC-3 prostate tumor (green channel) showing uptake of AF647-labeled CPMV-PEG-bombesin (heat map) over time. Images are representative of $n$ $=10$ experiments. Colors correspond to tumor/stroma ratio (see key). Scale bar $=3 \mathrm{~mm}$. B) Intravital imaging of PC-3 prostate tumor (green channel) showing uptake of AF647-labeled CPMV-PEG (heat map) over time. Images are representative of $\mathrm{n}=10$ experiments. Colors correspond to tumor/stroma ratio (see key). Scale bar $=3 \mathrm{~mm}$. C) Quantitation of tumor uptake of CPMV conjugates over time, $\mathrm{n}=10$ experiments per group. Values expressed as mean tumor/stroma ratio, using GFP channel to delineate tumor. Uptake of CPMV-PEGbombesin is significantly higher than CPMV PEG at $2 \mathrm{~h}$ and beyond $(\mathrm{P}<0.0001)$. D) Accumulation of CPMV conjugates in tumor tissue as assessed by fluorescence 
immunohistochemistry of frozen sections. Grayscale and color merged images are provided with PC-3 GFP cells (green) and CPMV-AF647 conjugates (red). Scale bar $=75 \mu \mathrm{m}$. 